\title{
Electrical restitution in the endocardium of the intact human right ventricle
}

\author{
John M Morgan, David Cunningham, Edward Rowland
}

\begin{abstract}
Objective-To characterise electrical restitution in the intact human heart.

Patients and Methods-A series of monophasic action potential electrical restitution curves were constructed from a single right ventricular endocardial site in eight patients (three men) without structural heart disease aged 52-68 (mean 55 years). A combination pacing/ monophasic action potential electrode was used to pace and record monophasic action potentials at drive cycle lengths of from $350 \mathrm{~ms}$ to $1500 \mathrm{~ms}$. Ventricular extrastimuli were delivered at 20 cycle intervals and decreased from the longest coupling interval attainable without escape beats.
\end{abstract}

Results-Restitution curves shifted downward and towards the left; steady state action potential duration shifted from the restitution plateau and descen-

Royal Brompton National Heart and Lung Hospital, London J M Morgan D Cunningham E Rowland

Correspondence to Dr John M Morgan, Royal Brompton National Heart and Lung Hospital, Sydney Street, Lydney Street, Accepted for publication 12 September 1991 to the mechanism of ventricular tachycardia induction by the delivery of a premature extrastimulus during clinical electrophysiology study, when action potential shortening and the altered velocity of conduc-
The action potential of a myocardial cell tion may contribute to the initiation of a reentry circuit. ${ }^{2-4}$

The electrical restitution curve relates the amount of action potential shortening to the prematurity of an extrastimulus. ${ }^{5}$ That relation has been characterised by the transmembrane action potential recording in in vitro preparations ${ }^{56}$ and has also been investigated in the intact human heart in vivo using the monophasic action potential..$^{7-9}$ In retrospect, these latter studies had drawbacks because investigators were unable to determine the duration of the monophasic action potential at the site of stimulation (so introducing conduction delay after premature ventricular extrastimulation which may have limited shortening of the diastolic time interval), ${ }^{7-9}$ because only one ${ }^{89}$ or two ${ }^{7}$ drive rates were used within individuals or because restitution curves were constructed with extrastimulus coupling intervals that were too long to chart accurately the sharp descent of the restitution curve. $^{8}$

The purpose of this investigation was to use a "combination" monophasic action potential recording/pacing electrode ${ }^{10}$ to characterise electrical restitution in the intact heart at a series of drive rates in a manner analogous to the characterisation of electrical restitution in single cell preparations. ${ }^{6}$ The clinical relevance of these observations is discussed.

\section{Patients and methods}

Eight patients (three male) aged 52-68 (mean 55 years) undergoing electrophysiology study of either atrioventricular reentrant tachycardia structural heart disease on echocardiography or exercise stress testing at high workload were recruited for this study. All patients gave informed consent for the study protocol which had been approved by the ethics committee of the National Heart and Chest Hospitals.

\section{TECHNIQUE}

Patients were studied in the resting postabsorptive unanaesthetised state. Femoral venous access was obtained by a modified Seldinger technique and two $6 \mathrm{~F}$ introducers were inserted into the right femoral vein. A combination electrode (EP Technologies, Mountain View, California, USA) to measure the monophasic action potential was passed to the right ventricle under fluoroscopic control. The position of the combination electrode was adjusted to give satisfactory monophasic action potential signals. The threshold for ded the curve, the amount of shift being linearly related to drive cycle length in two patients in whom the relation could be assessed; the amount of monophasic action potential shortening was a function of the degree of prematurity and that relation was unafiected by drive rate; the magnitude of restitution and the time constant of the restitution curve were not changed significantly by altered drive cycle length.

Conclusion-In the intact heart in vivo, electrical restitution (of the monophasic action potential) has similar characteristics to those (of the transmembrane action potential) in cellular preparations in vitro. Thus the alteration of action potential plateau currents by instantaneous rate change or drug effects, which can be directly observed by techniques available to the cellular electrophysiologist, may be indirectly assessed in vivo by characterisation of the effect of these on eiectrical restitution. or sinus node disease but without evidence of (1) achieves a constant duration when the frequency of its depolarisation is constant but shortens instantaneously if a premature 


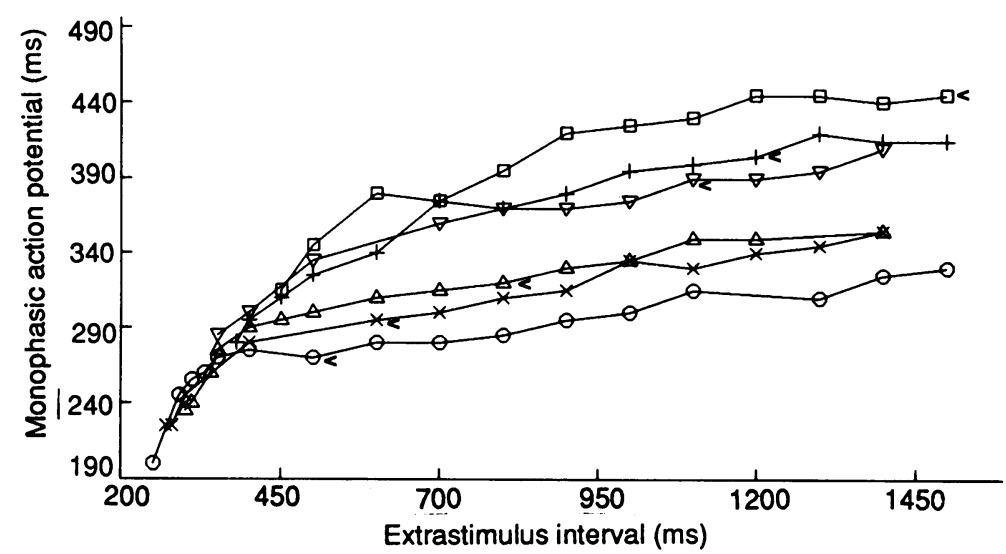

$*$ Drive cycle $1500 \mathrm{~ms} \quad$ + Drive cycle $1200 \mathrm{~ms} \quad *$ Drive cycle $1000 \mathrm{~ms}$
$*$ Drive cycle $750 \mathrm{~ms} \quad *$ Drive cycle $600 \mathrm{~ms} \quad \forall$ Drive cycle $600 \mathrm{~ms}$

Figure 1 A series of electrical restitution curves constructed at a right ventricular endocardial site; drive cycle lengths ranging from $500 \mathrm{~ms}$ to $1500 \mathrm{~ms}$.

ventricular pacing with the combination electrode was determined and ventricular pacing was performed at twice diastolic threshold with a programmed stimulator (Biotronik, Berlin, Germany). All monophasic action potential signals were recorded for analysis at a paper speed of $100 / 200 \mathrm{~mm} / \mathrm{s}$ on an inkjet recorder (Siemens Elema Mingograf, Sweden).

\section{STUDY PROTOCOL}

A series of electrical restitution curves was constructed in individuals at a single right ventricular endocardial site and at incremented drive cycle lengths. Ventricular pacing was performed for two minutes at each drive cycle length to establish a steady state. Thereafter, ventricular extrastimuli were delivered at twenty cycle intervals, reducing the coupling interval in decrements of $40 \mathrm{~ms}$, $20 \mathrm{~ms}$ and $10 \mathrm{~ms}$ from the drive cycle length until refractoriness was encountered. We confirmed that the twenty cycle interval between

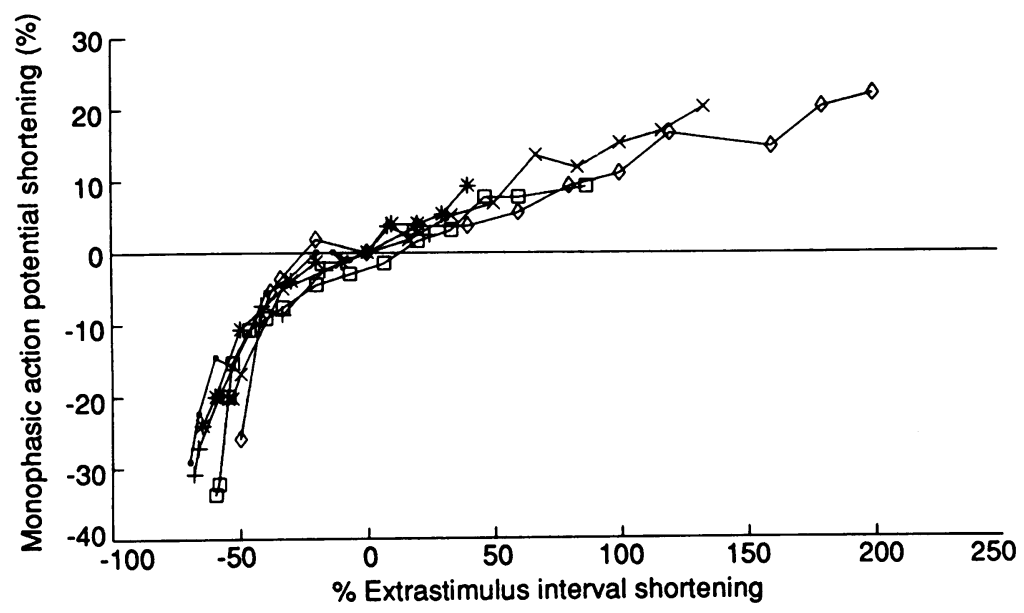

$$
\begin{array}{lll}
\text { - Drive cycle } 1500 \mathrm{~ms} & + \text { Drive cycle } 1200 \mathrm{~ms} & * \text { Drive cycle } 1000 \mathrm{~ms} \\
* \text { Drive cycle } 750 \mathrm{~ms} & * \text { Drive cycle } 600 \mathrm{~ms} & * \text { Drive cycle } 600 \mathrm{~ms}
\end{array}
$$

Figure 2 "Site-specific" restitution curve; percentage change in premature monophasic action potential duration from steady state monophasic action potential duration plotted against percentage change in extrastimulus coupling interval from drive cycle length for all electrical restitution curves shown in figure 1 . extrastimuli was adequate to allow maintenance of a steady state background upon which to deliver extrastimuli (despite perturbation by the premature stimulus) by measurement of the monophasic action potential duration preceding premature stimulation, which was found to remain constant throughout. Extrastimuli were also delivered at twenty cycle intervals with an increased coupling interval from the drive cycle length by allowing one second intervals between drive trains until escape beats occurred. In this way electrical restitution curves were constructed in a manner similar to that previously described in the investigation of electrical restitution in cellular preparations. ${ }^{56}$

\section{DATA AND STATISTICAL ANALYSIS}

Monophasic action potential durations were determined at $90 \%$ repolarisation. ${ }^{11}$ Magnitude of restitution was defined as the difference between the monophasic action potential duration elicited by the shortest extrastimulus coupling interval that elicited a propagated response and the monophasic action potential duration on the plateau of the electrical restitution curve. The maximum percentage shortening of the monophasic action potential from the steady state monophasic action potential duration was determined at each drive rate and compared within individuals. Electrical restitution curve time constants were obtained by fitting simple exponential curves to the electrical restitution curves at each drive rate. ${ }^{6}$

In all patients curves were constructed at $400 \mathrm{~ms}, 500 \mathrm{~ms}, 600 \mathrm{~ms}$, and $700 \mathrm{~ms}$ drive cycle lengths and differences in restitution curve magnitude, maximum percentage action potential shortening, and time constant of restitution were compared between these drive rates by the Kruskal-Wallis test and significance was taken as $\mathrm{p}<0.05$.

\section{Results}

Restitution curve series (4-7 curves) were obtained over a range of drive cycle lengths from $350 \mathrm{~ms}$ to $1500 \mathrm{~ms}$. In all patients' restitution curve series the following characteristics were identified.

Restitution curves shifted downwards and to the left with increasing drive rate (fig 1). Restitution curves were biphasic in all individuals, though the duration of the "supernormal" action potentials, and therefore the height of the restitution "hump", varied between individuals. However, in all instances it was small compared with the magnitude of the restitution curve $(<20 \%)$. There was also variation in the size of the restitution "hump" within individuals so that "supernormal" action potentials were longer at faster drive rates in some and at intermediate or slower drive rates in others.

For any given percentage decrease (from drive cycle length) of extrastimulus coupling interval there was a corresponding percentage shortening of monophasic action potential duration (from steady state duration) that was 
Table 1 Magnitude of restitution curves at various drive rates in eight patients

\begin{tabular}{|c|c|c|c|c|c|c|c|c|c|c|c|c|}
\hline \multirow[b]{2}{*}{ Patient } & \multicolumn{12}{|c|}{ Drive cycle length (ms) } \\
\hline & 1500 & 1200 & 1000 & 900 & 800 & 750 & 700 & 600 & 500 & 450 & 400 & 350 \\
\hline $\begin{array}{l}1 \\
2 \\
3 \\
4 \\
5 \\
6 \\
7 \\
8\end{array}$ & 130 & 135 & 125 & $\begin{array}{l}60 \\
85\end{array}$ & 45 & 135 & $\begin{array}{r}135 \\
35 \\
60 \\
40 \\
85 \\
110 \\
50 \\
90\end{array}$ & $\begin{array}{r}120 \\
55 \\
70 \\
50 \\
95 \\
100 \\
40 \\
85\end{array}$ & $\begin{array}{r}130 \\
55 \\
80 \\
70 \\
90 \\
90 \\
45 \\
95\end{array}$ & 40 & $\begin{array}{r}125 \\
40 \\
70 \\
45 \\
90 \\
110 \\
45 \\
100\end{array}$ & $\begin{array}{r}100 \\
50\end{array}$ \\
\hline
\end{tabular}

independent of drive rate and characteristic of a particular site (fig 2).

With increasing drive rate the steady state monophasic action potential duration shifted along the restitution curve plateau towards the shoulder of the curve, although the drive rates used were not sufficiently fast to cause the monophasic action potential duration to approach the sharp descent of the electrical restitution curve (fig 1 ).

The magnitude of the restitution curve did not change significantly with increasing drive rate within patients (table 1 ).

The maximum percentage shortening of monophasic action potential duration after the most premature extrastimulus that elicited a propagated response did not change significantly with increasing drive rate (table 2). Simple exponential curves were fitted to the electrical restitution curves (correlation coefficients ranging from 0.85 to 0.96 ) and the time constants of these were not significantly different within individuals (ranging from $0.5 \mathrm{~s}$ to $1.3 \mathrm{~s}$ ).

In two patients sufficient electrical restitution curves were constructed over an appropriate range of drive cycle lengths to allow determination of the relation of the difference between the steady state action potential duration and the duration of the monophasic action potentials on the electrical restitution curve plateau with drive cycle length. This relation was linear at shorter drive cycle lengths and terminated in a plateau at longer drive cycle lengths as the steady state action potential duration moved onto the restitution curve plateau from the shoulder of the restitution curve (fig 3 ).

\section{Discussion}

After Gettes et al described shortening of the action potential duration after premature extrastimulation, ${ }^{1}$ Bass characterised action

Table 2 Maximum percentage shortening from steady-state monophasic action potential duration during construction of electrical restitution curves at various drive rates in eight patients

\begin{tabular}{|c|c|c|c|c|c|c|c|c|c|c|c|c|}
\hline \multirow[b]{2}{*}{ Patient } & \multicolumn{12}{|c|}{ Drive cycle length (ms) } \\
\hline & 1500 & 1200 & 1000 & 900 & 800 & 750 & 700 & 600 & 500 & 450 & 400 & 350 \\
\hline $\begin{array}{l}1 \\
2 \\
3 \\
4 \\
5 \\
6 \\
7 \\
8\end{array}$ & 29 & 30 & 29 & $\begin{array}{l}22 \\
25\end{array}$ & 18 & 36 & $\begin{array}{l}35 \\
13 \\
16 \\
19 \\
33 \\
29 \\
11 \\
33\end{array}$ & $\begin{array}{r}25 \\
16 \\
23 \\
22 \\
41 \\
28 \\
8 \\
33\end{array}$ & $\begin{array}{r}32 \\
22 \\
24 \\
33 \\
41 \\
27 \\
8 \\
35\end{array}$ & 9 & $\begin{array}{r}35 \\
17 \\
23 \\
33 \\
45 \\
32 \\
9 \\
34\end{array}$ & $\begin{array}{l}38 \\
12\end{array}$ \\
\hline
\end{tabular}

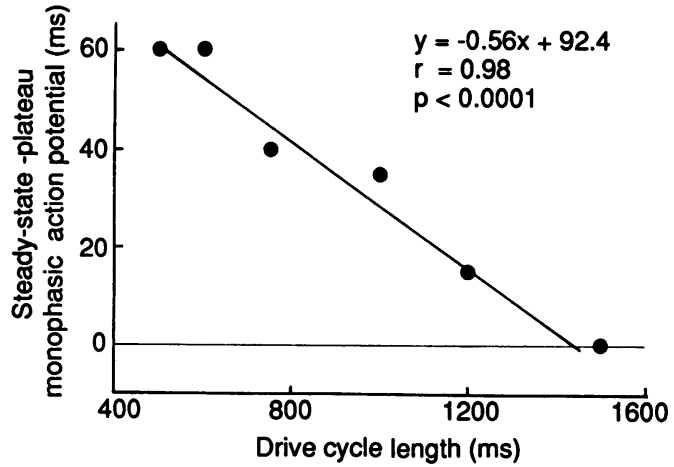

Figure 3 Plot of difference between steady state monophasic action potential duration and monophasic action potential duration on the plateau of the electrical restitution curve against drive cycle length for the series of electrical restitution curves shown in figure 1.

potential shortening after premature extrastimulation as electrical restitution ${ }^{5}$ and evidence has since been found that electrical restitution is determined by slow recovery of plateau currents from the processes of activation and inactivation ${ }^{12}{ }^{13}$ (although others have suggested that potassium accumulation in the extracellular clefts may also have a role. ${ }^{14}$

The influence of drive rate on electrical restitution in cellular preparations has been characterised by Boyett and Jewell ${ }^{5}$ with further observations made by Boyett and Fedida ${ }^{15}$ who have shown that an increase in drive rate causes the electrical restitution curve to shift down and to the left: down because the steady state action potential has adapted at a higher rate to a shorter duration and to the left because incomplete recovery of the action potential plateau currents causes the steady state action potential duration to move from the restitution curve plateau towards the shoulder of the curve.

Franz and colleagues ${ }^{78}$ and Seed and colleagues $^{9}$ used the monophasic action potential recording technique to investigate electrical restitution in the intact human heart. Both groups demonstrated shortening of the monophasic action potential after premature ventricular extrastimulation. Franz and colleagues found that an increase in drive rate caused electrical restitution curves to shift to the left and downwards as in cellular preparations ${ }^{515}$ but they compared only two drive rates and performed ventricular pacing in only two patients. $^{7}$ They also reported that electrical restitution curves could be biphasic with a phase of "supernormal" action potentials before the descent of the restitution curve and repeated this observation in a later report. ${ }^{8}$ "Supernormal" action potentials have also been noted on transmembrane action potential recordings from preparations of both human and animal hearts ${ }^{516-19}$ but not in other species ${ }^{613}$ and it is suggested there may be species differences. ${ }^{2021}$ Possible mechanisms of "supernormal" action potentials have been suggested. Bass ${ }^{5}$ and Greenspan and colleagues ${ }^{16}$ have suggested that a low calcium transient, caused by incomplete mechanical restitution after premature extrastimulation, results in increased inward calcium currents 
which maintain the action potential plateau and therefore prolong action potential duration. Iinuma and $\mathrm{Kato}^{22}$ reported that calcium channel blocking agents suppress supernormal restitution and this is cited as evidence for this "calcium theory". The situation is almost certainly more complex because the principal inward current supporting the action potential plateau may be the sodium/calcium exchange current and not inward movement of calcium. ${ }^{23}$ However, in the presence of a low internal calcium transient, the fast inward calcium current may be greater in amplitude and duration $^{24}$ and could therefore support a longer action potential duration during the early restitution phase than that determined by recovery of plateau currents alone. However, Seed and colleagues did not find that electrical restitution was biphasic ${ }^{9}$ but this may have been because of the size of the decremented coupling interval that was used to plot the electrical restitution curve.

We found that the restitution curve is displaced downwards and to the left at faster drive rates and that the steady state action potential duration moves towards the shoulder of the restitution curve. However, in contrast to the observations of Boyett and Jewell, ${ }^{6}$ the difference in the magnitude of the restitution curve did not decrease significantly with increasing drive rate. Similarly, the time constants of the restitution curves did not change significantly with increasing drive rate in this work whereas Boyett and Jewell reported an increase in the time constant with increasing drive rate. ${ }^{6}$ However, the range of drive rates (minimum $350 \mathrm{~ms}$ and maximum $1500 \mathrm{~ms}$ drive cycle length) that could be used in this in vivo study in the intact human heart was narrower of necessity than that used in the investigation of electrical restitution in vitro (from 0.25 beats/ min to 220 beats/min) and this constraint may explain these discordant observations. Also a phase of "supernormal" restitution was observed immediately after the plateau phase in all curves and in all patients although the increase in monophasic action potential duration varied with drive rate within and between individuals. This suggests that the phenomenon is influenced by the degree of prematurity, the background drive rate, and perhaps other site-specific factors.

We suggest that these observations are relevant to clinical electrophysiology because ventricular tachycardia study protocols use premature ventricular extrastimulation during ventricular drive pacing to induce ventricular arrhythmia. ${ }^{2526}$ A suggested mechanism of ventricular tachycardia induction after premature ventricular extrastimulation is creation of a reentry circuit due to slowed conduction, dispersion of refractoriness, or a combination of both. $^{2-4}$ There is experimental evidence that altered dispersion of refractoriness or action potential duration contributes to the arrhythmogenic substrate. Kuo and colleagues used a "global hypothermia with regional warm blood perfusion model" to induce disorganisation of repolarisation and observed a critical exaggeration of regional variation in monophasic action potential duration which allowed the induction of sustained ventricular tachycardia with a single extrastimulus. ${ }^{27}$ The same group showed in later work that dispersion of the monophasic action potential duration was further exaggerated by a premature extrastimulus though a second premature extrastimulus was required for the induction of ventricular arrhythmia. ${ }^{28}$ In earlier work, Han and Moe reported that temporal dispersion of recovery of excitability was minimal in steady state conditions but increased after premature extrastimulation. ${ }^{29}$ In the clinical context, it has been suggested that dispersion of refractoriness creates the substrate for arrhythmia, after premature ventricular extrastimulation ${ }^{30}$ or a premature extrasystole succeeding a "short-long" sequence. $^{31}$ Our own group has also found evidence of abnormal electrical restitution in diseased myocardium. ${ }^{32}$

Thus characterisation of electrical restitution in normal and diseased myocardium may give insight into the contribution of cellular mechanisms to the arrhythmogenic substrate. Furthermore, the drug effect on electrical restitution may characterise some aspects of drug antiarrhythmic properties and recent reports have described the effect of new class II agents on action potential plateau currents. ${ }^{33}$ The drug effect on electrical restitution may therefore offer unique opportunities to determine drug action both in vitro and during clinical electrophysiology study in vivo.

In conclusion, electrical restitution in the intact heart (investigated by the monophasic action potential recording technique) resembles electrical restitution in cellular preparations. This may offer a bridge between clinical and cellular electrophysiology, allowing the observations of the cellular electrophysiologist on action potential plateau currents in vitro to be explored in clinical studies in vivo.

1 Gettes LS, Morehouse N, Surawicz B. Effects of premature depolarisation on the duration of action potentials in Purkinje fibers of the moderator band of the pig heart. Role of the proximity and the duration of the preceding action potential. Circ Res 1972;30:55-66.

2 Wellens HJJ, Duren DIE, Lie LI. Observations on the mechanisms of ventricular tachycardia in man. Circulation mechanisms of vent

3 Wit AL, Rosen MR. Pathophysiologic mechanisms of cardiac arrhythmias. Am Heart J 1983;106:798-811.

4 Josephson ME, Marchlinski ME, Buxton AE, Waxman HL Doherty JU, Kienzle MG, et al. Electrophysiological basis for sustained ventricular tachycardia-role of reentry. In Josephson ME, Wellens HJJ, eds. Tachycardias: mechanisms, diagnosis, treatment. Philadelphia: Lea and Febiger, 1984:305-52.

5 Bass BG. Restitution of the action potential in cat papillary muscle. Am J Physiol 1975;228:1717-24.

6 Boyett MR, Jewell BR. A study of the factors responsible for rate-dependent shortening of the action potential in mammalian ventricular muscle. J Physiol (Lond) 1978; 285:359-80.

7 Franz MR, Schaefer J, Schottler M, Seed WA, Noble MIM. Electrical and mechanical restitution of the human heart at
different rates of stimulation. Circ Res 1983;53:815-22.

8 Franz MR, Swerdlow CD, Bing Liem L, Schaefer J. Cycle length dependence of human action potential duration in length dependence of human action
vivo. $J$ Clin Invest 1988;82:173-80.

9 Seed WA, Noble MIM, Oldershaw P, Wanless RB, DrakeHolland AJ, Redwood D, et al. Relation of human cardiac action potential duration to the interval between beats: implications for the validity of rate
(QTc). Br Heart J 1987;57:32-7.

10 Franz MR, Chin MC, Sharkey HR, Griffin JC, Scheinman MM. A new single catheter technique for simultaneous MM. A new single catheter technique for simultaneous period in vivo. J Am Coll Cardiol 1990;16:878-86. 
11 Franz MR, BurkhoffD, Spurgeon H, Weisfeldt ML, Lakatta EG. In vitro validation of a new cardiac catheter technique for recording

2 Hauswirth $\mathrm{O}$, Singh $\mathrm{BN}$. Ionic mechanisms in heart muscle in relation to the genesis and the pharmacological control in relation to the genesis and the pharmacological con

13 Gettes LS, Reuter H. Slow recovery from inactivation of inward currents in mammalian myocardial fibres. JPhysiol (Lond) 1974;240:703-24.

14 Kline RP, Kupersmith J. Effects of extracellular potassium accumulation and sodium pump activation on automatic canine Purkinje fibres. J Physiol 1982;324:507-33.

15 Boyett MR, Fedida D. Changes in the electrical activity of dog cardiac Purkinje fibres at high heart rates. $J$ Physiol 1984;350:361-91.

16 Greenspan K, Edmands RE, Fisch C. Effects of cycle-length alteration on canine cardiac action potentials. Am JPhysiol 1967;212:1416-20.

17 Edmands RE, Greenspan K, Fisch C, Anderson GJ. Correlation between the monophasic action potential and the inotropic response of human myocardium. Circulation 1967;36:101-18.

18 Miller JP, Wallace AG, Feezor MD. A quantitative comparison of the relation between the shape of the action potential and the pattern of stimulation in canine venpotential and the pattern of stimulation in canine ventricular muscle

19 Noble $D$, Cohen $I$. The interpretation of the $T$ wave of the electrocardiogram. Cardiovasc Res 1978;12:13-27.

20 Wohlfart B. Relationships between peak force, action potential duration and stimulus interval in rabbit myocardium. Acta Med Scand 1979;106:395-409.

21 Elzinga G, Lab MJ, Noble MIM, Papadoyannis DE Pidgeon J, Seed A, et al. The action potential duration and contractile response of the intact heart related to the preceding interval and the preceding beat in the dog and cat. J Physiol (Lond) 1981;313:481-500.

22 Iunuma $H$, Kato $K$. Mechanisms of augmented premature responses in canine ventricular muscle. Circ Res 1979; 44:624-9.

23 Egan T, Noble D, Noble SJ, Powell T, Spindler AJ, Twist VW. Sodium-calcium exchange during the action poten- tial in guinea-pig ventricular cells. $J$ Physiol 1989;411: 639-61.

24 Hilgemann DW, Noble D. Excitation-contraction coupling and extracellular calcium transients in rabbit atrium. casic cellular mechanisms. Proc $R$ Soc Lond 1987;230:163-205.

25 Waldo AL Akhtar M, Brugada $P$, Henthorn RW, Scheinaldo Al, Akhtar $M$, Brugada $P$, Henthom man MM, Ward DE, et al. NASPE policy statement: the minimally appropriate electrophysiologic study for the initial assessment of patients with documented sustained monomorphic ventricular tachycardia. $P A C E$ 1985;

26 Wellens HJJ, Schuilenburg RM, Durrer D. Electrical stimulation of the heart in patients with ventricular tachycardias. Circulation 1972;46:216-26.

27 Kuo CS, Munakata K, Reddy CP, Surawicz B. Characteristics and possible mechanisms of ventricular arrhythmia dependent on the dispersion of action potential durations. Circulation 1983;67:1356-67.

28 Kuo CS, Atarashi H, Reddy CP, Surawicz B. Dispersion of ventricular repolarisation and arrhythmia: study of two consecutive ventricular premature complexes. Circulation $1985 ; 72: 370-6$

29 Han J, Moe GK. Nonuniform recovery of excitability in ventricular muscle. Circ Res 1964;14:44-60.

30 Mitchell LB, Wyse DG, Duff HJ. Programmed electrical stimulation for ventricular tachycardia induction in stimulation for ventricular tachycardia induction in hess in tachycardia induction. J Am Coll Cardiol 1986; ness in tach

31 Gomes JA, Alexopoulous D, Winters SL, Deshmukh P, Fuster V, Suh $K$. The role of silent ischemia, the arrhythmia substrate and the short-long sequence in the genesis of sudden cardiac death. J Am Coll Cardiol 1989;14:377-84.

32 Morgan JM, Cunningham AD, Rickards AF, Rowland E. Exaggerated dispersion of repolarisation provoked by premature ventricular stimulation: a mechanism for ventricular arrhythmia [abstract]. Br Heart J 1990;64:87.

33 Sanguinetti MC, Jurkiewicz NK. Two components of cardiacdelayed rectifier $\mathrm{K}^{+}$current:Differential sensitivity to block by Class III antiarrhythmic agents. J Gen Physiol 1990;96:77-84. 